\title{
ORIGINAL
}

\section{FIEBRE BOTONOSA MEDITERRÁNEA: EPIDEMIOLOGÍA EN ESPAÑA DURANTE EL PERIODO 2009-2012}

\author{
Belén García-Magallón (1), María Cuenca-Torres (2), Flor Gimeno-Vilarrasa (2) y Antonio Guerrero- \\ Espejo (1,2).
}

(1) Facultad de Medicina, Universidad Católica de Valencia.

(2) Dirección de Investigación y Docencia. Hospital Universitario de la Ribera. Alzira (Valencia)

\section{RESUMEN}

Fundamento: La Fiebre Botonosa Mediterránea (FBM) es una zoonosis producida por Rickettsia conorii cuyo vector es Rhipicephallus sanguineus. El objetivo de este trabajo fue describir la epidemiología y el coste medio de la fiebre botonosa en España y sus comunidades autónomas durante el período 2009-2012)

Métodos: Se realizó un estudio observacional del período 2009-2012 utilizando como fuente de información el Conjunto Mínimo de Datos (CMBD), identificando las altas hospitalaria cuyo diagnóstico, de acuerdo a la Clasificación Internacional de Enfermedades $9^{\mathrm{a}}$ revisión Modificación Clínica (CIE-9MC) fue 82.1 (fiebre botonosa mediterránea). Se calcularon las tasas de incidencia de dicha enfermedad en España y cada una de las comunidades autónomas. El coste medio (en euros) se calculó de acuerdo a la norma estatal para grupos diagnósticos relacionados. Se utilizó el software fStats para la comparación de tasas según la edad, sexo, distribución anual, estacional y por CCAA.

Resultados: Durante el período de estudio se identificaron 667 ingresos con diagnóstico al alta de fiebre botonosa mediterránea, siendo la tasa de incidencia fue de 0,36 casos por 100.000 habitantes. La mayor incidencia se dio en Ceuta y La Rioja con una incidencia de 1,9 y 1,87 casos por 100.000 habitantes y año. No se detectaron casos en Cantabria ni en Canarias. El riesgo relativo hombre/mujer fue 2:1 (valor de $p<0,05$ ). El grupo etario predominante fue el de los mayores de 55 años ( 327 casos). Los meses con más casos fueron de junio-septiembre (466 casos), produciéndose un pico de casos en agosto (137 casos). La tasa de mortalidad fue $0,3 \%$. El coste medio fue de $4.647,205 €$

Conclusión: La incidencia de la fiebre botonosa mediterránea durante el período estudiado fue menor que en períodos anteriores, con una distribución por comunidades autónomas heterogénea y con mayor frecuencia durante el mes de agosto. Se detectaron pacientes de todas las edades, predominando los varones mayores de 55 años. La tasa de mortalidad intrahospitalaria fue pequeña.

Palabras clave: Incidencia. Fiebre botonosa mediterránea. España. Fiebre exantemática mediterránea. Rickettsia conorii.

\section{ABSTRACT Mediterranean Spotted Fever: Epidemiological Assessment in Spain during the Period 2009-2012}

Background: The Mediterranean Spotted Fever (MSF) is a zoonosis, produced by Rickettsia conorii whose vector is Rhipicephallus sanguineus. The aim of this study was to describe the epidemiology in Spain and its Autonomous Communities (AA.CC) and the average cost during the period 2009-2012.

Methods: We conducted a retrospective observational study of patients, between 2009-2012, whose diagnostic at hospital discharge was encoded, according to the International Classification of Diseases 9th revision Clinical Modification (ICD-9CM) as 82.1 (MSF). The information was collected through the minimum basic data set. Incidence rates of the disease were calculated in Spain and its Autonomous Communities. The fStats software was used for comparison of rates based on age, sex, annual, seasonal and AA.CC's distribution. The average cost (in euros) was calculated according to the state standard

Results: The incidence rate was 0,36 cases per 100,000 inhabitants and year during 2009-2012, with 667 admissions. The highest incidence was obtained in Ceuta and La Rioja with an incidence of 1,9 and 1,87 cases per 100,000 inhabitants per year. No cases were detected in Cantabria nor Canarias. The relative risk male female was $2: 1$ ( $p$ value $<0,05$ ). The predominant age group was over 55 years ( 327 cases). The months with the most cases were from June to September (466 cases), producing a peak in the number of cases in August (137 cases). The mortality rate was 0,3\%. The average cost was $4.647,205 €$.

Conclusion: The incidence of MSF was low, with a heterogeneous geographical distribution and with higher frequency during the month of August. Patients of all ages were detected, predominantly for males over 55 years old. The hospital mortality rate was small.

Key words: Incidence. Mediterranean spotted fever. Spain. Rickettsia conorii.

Correspondencia

Belén García Magallón.

C/ Jarama, 14

Majadahonda

28220 Madrid

belengarciamag@hotmail.es

DOI: 


\section{INTRODUCCIÓN}

La Fiebre Botonosa Mediterránea (FBM) es una zoonosis incluida en el grupo de las rickettsiosis humanas. El género Rickettsia comprende diferentes especies de bacterias cocobacilos gramnegativos, parásitos intracelulares obligados $^{1}$. La FBM, producida por Rickettsia conorii es endémica en el sur de Europa y en la mayoría de los países ribereños del Mediterráneo, Mar Negro y Mar Caspio ${ }^{2}$. En la cuenca mediterránea, el vector principal de la FBM es la garrapata del perro (Riphicephallus sanguineus) ${ }^{1}$.

La enfermedad fue descrita por primera vez en 1910 en Túnez ${ }^{3}$, aunque hasta 1932 no se conoció el papel de $R$. sanguineus como vector ${ }^{4}$. La enfermedad sufrió una gran expansión, a veces de forma epidémica, durante las décadas de 1930 y 1940, disminuyendo posteriormente. En los años sesenta y buena parte de los ochenta resurgió en los países de la cuenca mediterránea (España, Francia, Italia e Israel), donde es considerada una enfermedad endémi$\mathrm{ca}^{1}$. La FBM no fue incluida como enfermedad de declaración obligatoria a nivel nacional hasta $1996^{5}$ y a partir de ese año continuó como enfermedad de declaración obligatoria sólo en algunas comunidades autónomas.

El diagnóstico se basa en la clínica, serología y epidemiología. La enfermedad afecta a ambos sexos, con un ligero predominio en los hombres y es más frecuente en las dos primeras décadas de vida ${ }^{1}$.

El patrón temporal de la FBM es estacional, aunque en España pueden detectarse casos durante todo el año, pero es más frecuente en verano y principios de otoño, igual que en otras zonas de la Unión Europea con climas templados, siguiendo los periodos de actividad de los vectores que la transmiten ${ }^{2}$.

La enfermedad suele ser leve aunque puede tener un curso grave en pacientes con factores de riesgo como diabetes mellitus, insuficiencia cardiaca, alcoholismo, ancianos o déficit de G6PD ${ }^{2}$.
El objetivo principal de este trabajo fue realizar una estimación de la incidencia en España y sus comunidades autónomas entre los años 2009-2012.

\section{MATERIAL Y MÉTODO}

Se diseñó un estudio observacional utilizando como fuente de información el Conjunto Mínimo Básico de Datos (CMBD) del Sistema Nacional de Vigilancia Epidemiológica para ingresos hospitalarios, gestionado por el Ministerio de Sanidad, Servicios Sociales e Igualdad. El CMBD consiste en un registro de variables demográficas y sanitarias de las personas ingresadas en los hospitales. Utiliza los códigos clínicos de la versión española de la novena Clasificación Internacional de Enfermedades $9^{a}$ revisión, modificación clínica (CIE-9-MC) ${ }^{6}$.

Para esta investigación se analizaron datos de todos los ingresos hospitalarios cuyo diagnóstico principal o secundarios al alta hospitalaria se hubiera codificado en el CMBD, como 082.1 (fiebre botonosa mediterránea). Los criterios diagnósticos fueron basado en el juicio clínico. En el cálculo de la incidencia no se contabilizó a los pacientes que reingresaron por el mismo episodio de la enfermedad o cuando en un mismo paciente apareció por segunda vez con diagnóstico secundario de FBM en otro ingreso. La población del denominador fue la correspondiente a España y sus CCAA durante el periodo 2009-2012, obtenida del censo poblacional español a través del Instituto Nacional de Estadística?.

Los datos obtenidos fueron codificados e introducidos en una base de datos Excel y analizados a través de software fStats. Se realizó un análisis de frecuencia de los casos, calculándose estadísticos de medida de posición o tendencia central y de dispersión para la edad de las personas afectadas. Se determinó la incidencia por sexo utilizando como denominador la población de cada sexo en España ${ }^{7}$. Se calculó la incidencia anual de ingresos en España y por CCAA durante el 
periodo de estudio 2009-2012. Se valoró la incidencia según el sexo y la tasa de mortalidad. Se determinó la mediana de estancia y el coste medio.

Para la comparación de tasas independientes en función del sexo, distribución anual y por CCAA se utilizó el software fStats. Se consideró estadísticamentes significativos valores de $\mathrm{p}<0,05$.

El coste medio se calculó, de acuerdo a la norma estatal para el registro de altas de los hospitales generales del Sistema Nacional de Salud, como el coste medio en euros de los casos teniendo en cuenta los Grupos Relacionados por el Diagnóstico. Se calculó también su desviación estándar.

\section{RESULTADOS}

Durante 2009-2012 se obtuvieron 667 registros con el diagnostico de FBM. Un total de $596(89,5 \%)$ se registraron como diagnóstico principal y $71(10,5 \%)$ como diagnósticos secundarios. Medicina interna ingresó a $476(71 \%)$ pacientes, seguido de enfermedades infecciosas con $79(12 \%)$ y pediatría con $73(11 \%)$ pacientes .

En 2009 y 2010 se diagnosticaron 153 casos; en 2011196 casos y en 2012 165. La media de casos del periodo fue de 165 por año. La incidencia se situó en 0,36 casos por 100.000 habitantes y año en el periodo de 2009-2012. La mayor incidencia tuvo lugar en 2011 (0,42 casos por 100.000 habitantes) comparadas con las del 2009 y $2010(0,33$ casos por 100.000 habitantes) siendo las diferencias estadísticamente significativas, pero no lo fue respecto a la incidencia del 2012 (0,35 casos por 100.000 habitantes) (tabla 1$)$.

La comunidad autónoma con mayor número de casos fue Andalucía con 186 (28\%), seguida de Cataluña con 89 (13,34\%) (tabla 2). La tasa de incidencia anual por 100.000 habitantes fue significativamenete mayor en Ceuta y La Rioja (1,9 y 1,87 respectivamente) en comparación con el resto de las CC.AA

\begin{tabular}{|c|c|c|c|}
\hline \multicolumn{4}{|c|}{ Tabla 1 } \\
\multicolumn{4}{|c|}{ Incidencia de FBM por 100.000 } \\
habitantes según año de estudio
\end{tabular}

\begin{tabular}{|l|r|r|r|}
\hline \multicolumn{4}{|c|}{ Tabla 2} \\
Incidencia por 100.000 habitantes \\
de fiebre botonosa mediterránea en \\
España durante el período 2009-2011 \\
\hline \multicolumn{1}{|c|}{ Provincia } & Casos & Población & $\begin{array}{c}\text { Incidencia } \\
\text { por 105 hab } \\
\text { y año }\end{array}$ \\
\hline Andalucía & 186 & 8.302 .923 & 0,56 \\
\hline Aragón & 7 & 1.345 .473 & 0,13 \\
\hline Asturias & 1 & 1.085 .289 & 0,02 \\
\hline Baleares & 29 & 1.095 .426 & 0,66 \\
\hline Canarias & 0 & 2.103 .992 & 0 \\
\hline Cantabria & 0 & 589.235 & 0 \\
\hline Castilla La Mancha & 48 & 2.081 .313 & 0,58 \\
\hline Cast y Leon & 38 & 2.563 .521 & 0,37 \\
\hline Cataluña & 89 & 7.475 .420 & 0,3 \\
\hline Ceuta & 6 & 78.674 & 1,9 \\
\hline C.valenciana & 62 & 5.094 .675 & 0,3 \\
\hline Extrematura & 28 & 1.102 .410 & 0,63 \\
\hline Galicia & 10 & 2.796 .089 & 0,1 \\
\hline Madrid & 62 & 6.386 .932 & 0,24 \\
\hline Melilla & 4 & 73.460 & 1,36 \\
\hline Murcia & 21 & 1.446 .520 & 0,8 \\
\hline Navarra & 6 & 2.172 .175 & 0,07 \\
\hline País vasco & 667 & 46.745 .807 & 0,36 \\
\hline La Rioja & & 1,87 \\
\hline España & & \\
\hline
\end{tabular}

excepto Melilla $(1,36)$, Navarra $(0,83)$ y Murcia $(0,8)$. En Cantabria y Canarias no se detectó ningún caso durante el período 20092012 (figura 1).

Del total de pacientes, 434 (65\%) fueron hombres y 233 (35\%) mujeres. La mayor incidencia de la enfermedad en el sexo masculino se detectó en La Rioja, con una tasa de 2,62 casos por 100.000 habitantes y año, y del sexo femenino en Ceuta con una inciden- 


\section{Figura 1}

Tasas de incidencia por 100.000 habitantes y año de fiebre botonosa en España y las comunidades autónomas durante 2009-2012

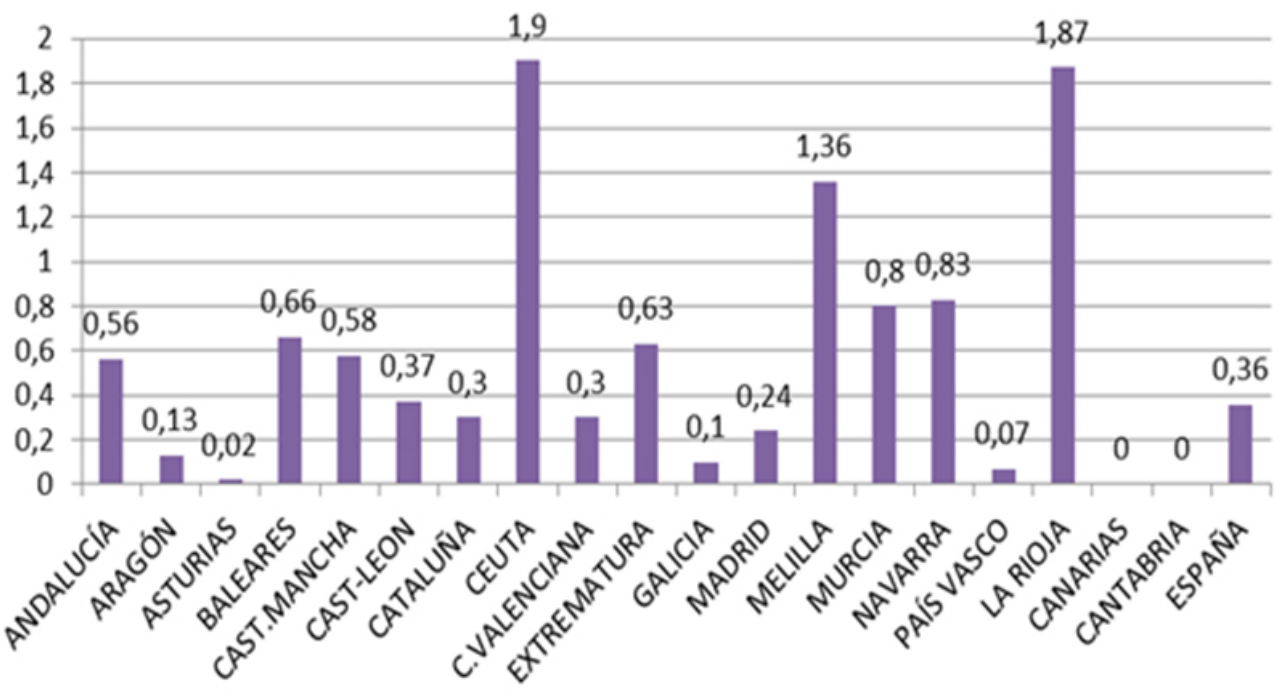

Figura 2

Distribución de casos de fiebre botonosa mediterránea por edad y sexo durante 2009-2012

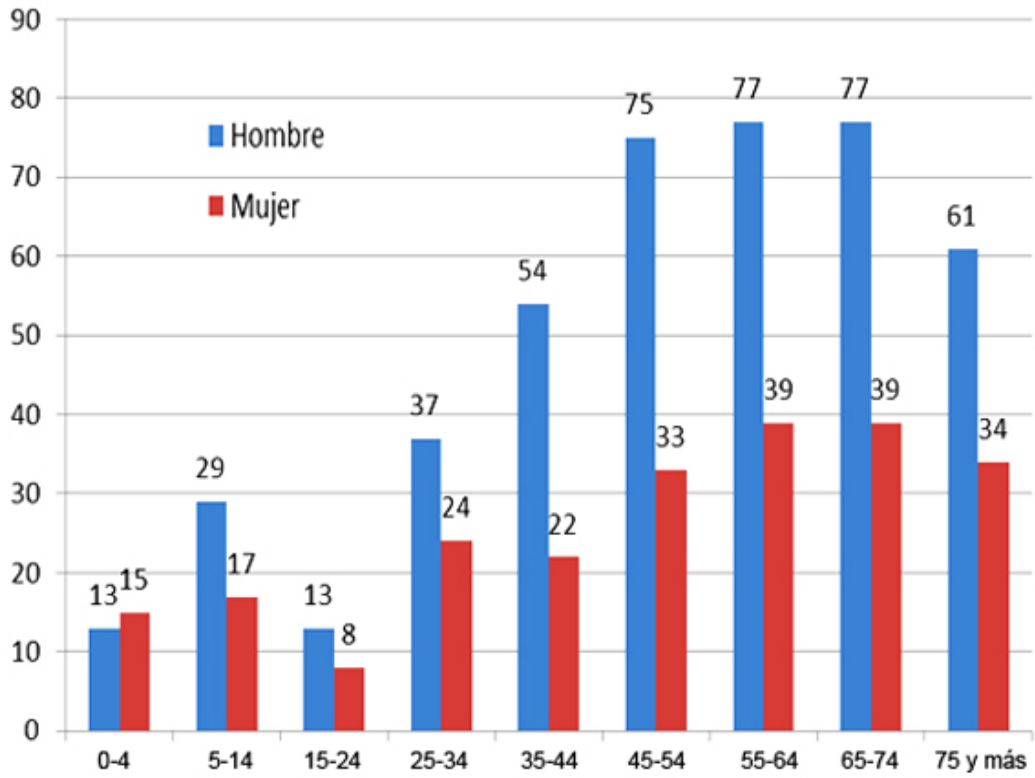


Figura 3

Porcentaje de personas ingresadas a causa de fiebre botonosa mediterránea durante 2009-2012 según el mes del año

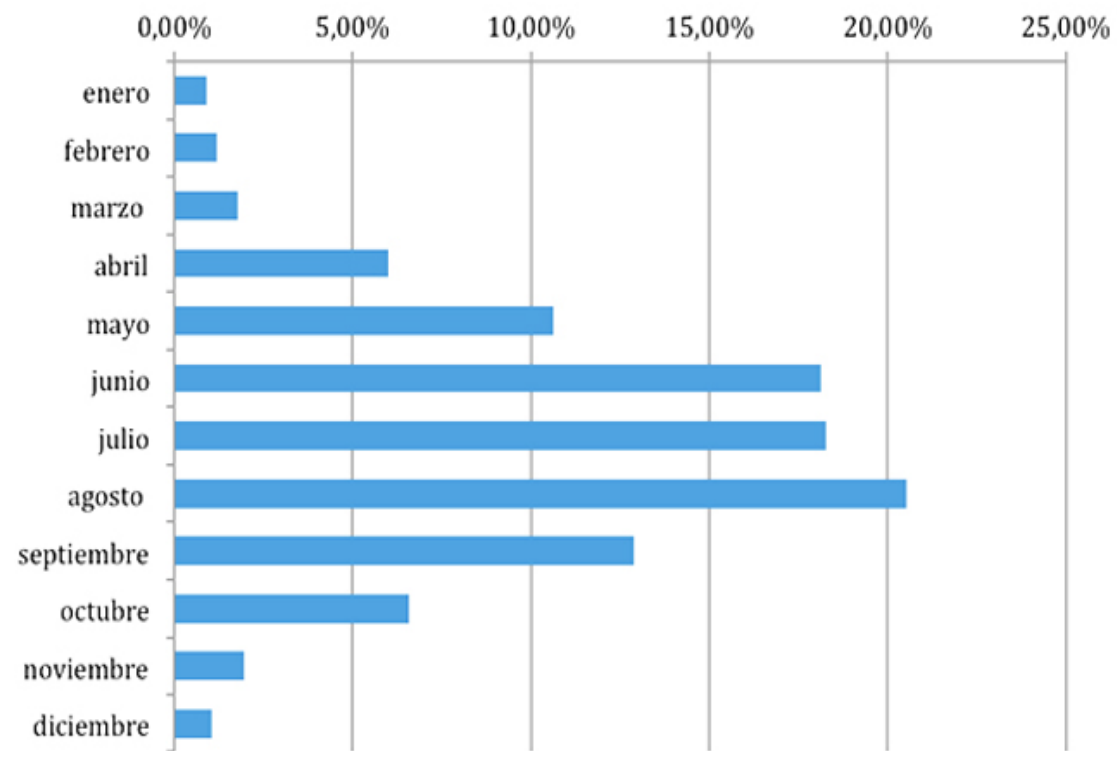

cia anual de 2,6 casos por 100.000 habitantes $\mathrm{y}$ año. La tasa de incidencia para hombres y mujeres fue de 0,36 y 0,25 por 100.000 habitantes y año respectivamente en España, durante el periodo de estudio. El riesgo relativo (RR) hombre-mujer fue 2:1 (IC95\%: 1,62; 2,23); $<<0,001$.

El grupo etario predominante correspondió a las personas mayores de 55 años con 327 (49\%) casos. En oposición, el grupo etario con menos casos diagnosticados fue el de 15-24 años con 21 (3,15\%). El único grupo etario donde hubo más casos en mujeres que en hombres fue el comprendido entre los 0-4 años (figura 2).

La media de edad de las personas afectadas fue de 50,49 años con una desviación típica de 22,65. La mediana de edad fue de 54 años con un rango de 0-91 años. La moda fue de 64 años.
Hubo ingresos todos lo meses del año. Agosto destacó con 137 (20\%) seguido de julio y junio, con 122 y 121 casos respectivamente $(18 \%)$. Se encontraron diferencias estadísticamente significativas entre las estaciones, con un claro predominio durante el verano y una clara disminución del número de casos durante los meses más fríos (figura 3).

Dos pacientes fallecieron durante el ingreso, ambos varones y diabéticos tipo II, de 59 y 61 años. El diagnóstico principal de los fallecidos fue fiebre botonosa mediterránea en uno y en el otro de endocarditis bacteriana aguda (diagnóstico secundario FBM). La tasa bruta de mortalidad intrahospitalaria fue del $0,3 \%$.

La mitad de los pacientes permanecieron ingresados más de 5 días (rango 1-73 días).

El coste medio fue de 4.647,205€ con una desviación estándar de 2.546,066. 


\section{DISCUSIÓN}

La tasa de incidencia de FBM durante el periodo 2009-2012 en el territorio español es menor que la última disponible de 1996 que fue de 1,36 casos por 100.000 habitantes y año ${ }^{8}$.

En otros países de la Unión Europea la situación es variable. Por ejemplo, en Croacia (periodo 1982-2002), la incidencia anual a través de diagnósticos hospitalarios fue de 1,27 casos por 100.000 habitantes $^{9} \mathrm{y}$ en Bulgaria se comunicó una reemergencia de casos, con una incidencia de 7 casos por 100.000 habitantes en $2011^{10}$. La mayor incidencia en España tuvo lugar en 2011 con 0,42 casos por 100.000 habitantes. Parece que la FBM tiene incidencias fluctuantes en el tiempo ${ }^{11}$. Nuestra incidencia, basada en los ingresos hospitalarios, puede infravalorar la incidencia real al no contabilizar la presentación casos con curso benigno y tratada ambulatoriamente. Cuando las enfermedades se analizan de manera integral, considerando atención primaria y especializada, la incidencia puede variar. Así, en la Comunidad Valenciana (CV) se estimó una incidencia de 0,52 casos por 100.000 habitantes y año a través de un sistema de vigilancia epidemiológica que incluye también los casos de FBM de atención primaria $^{12}$, mientras que en el mismo periodo nuestra incidencia fue de 0,36 casos por 100.000 habitantes y año. El TIBOLA/DEBONEL, otra rikettsiosis, también es más frecuente que la fiebre botonosa mediterránea al analizarlo de forma integral ${ }^{13}$.

La FBM es una enfermedad endémica sin significativas oscilaciones de incidencia en algunas zonas de España y emergente en otras $^{14-17}$. La FBM tiene una incidencia heterogénea entre sus diferentes CCAA. La variabilidad geográfica dentro del territorio español, puede venir condicionada por el grado de sospecha diagnóstica o por factores epidemiológicos, como el clima o la vegetación, determinantes en la transmisión del agente patógeno. Esto puede cambiar si el vector y el reservorio de la enfermedad lo hacen, tal vez por cambios climáticos, deforestaciones o migraciones de seres humanos y animales. Asimismo, las razones para la distribución de algunas enfermedades transmitidas por garrapatas aún no se han determinado, ya que $R$. Sanguineus se distribuye mundialmente ${ }^{18}$.

El Sistema de Vigilancia Epidemiológica (SVE) notificó que la mayor incidencia de FBM en las CCAA españolas se dio en La Rioja en el $2010^{15}$ y en el $2011^{16}$, lo que coincide con los resultados del presente estudio.

Un estudio realizado en la provincia de Albacete, que tenía en cuenta criterios clínicos y serológicos de pacientes (no solo de ingresos), reveló una tasa de incidencia de 1,6 casos por 100.000 habitantes y año durante el periodo de $1997-2003^{19}$, significativamente mayor que la obtenida en el presente trabajo en dicha provincia para el periodo 2009-2012 ( 0,68 casos por 100.000 habitantes y año).

La incidencia en este estudio fue mayor en hombres con cifras muy similares a las aportadas por un estudio realizado en Croacia ${ }^{9}$. En los datos aportados por el SVE durante el período 2009-2012 el predominio de casos fue mayor en la población masculina ${ }^{14-17}$. La mayor incidencia en hombres puede deberse a factores que influyen en las tasas de infecciones humanas transmitidas por garrapatas, como factores relacionados con el huésped: la probabilidad de que las personas estén en contacto con la garrapata (la mayor parte de agricultores en España son hombres) o la susceptibilidad de género ${ }^{18}$.

La mayor incidencia en la época estival coincide con otros estudios de diferentes países europeos ${ }^{9,10,19,20}$ y con los datos aportados por el SVE ${ }^{14-17}$. El mayor número de casos en la estación estival coincide con la mayor actividad de transmisión vectorial de la garrapata.

Las tasas de mortalidad antes de la era antibiótica se situaban entre el 1-3\%, habiendo descendido por debajo del $1 \%{ }^{10}$, como ocurre con la tasa hallada en el presente trabajo $(0,3 \%)$. 
Las limitaciones principales de este trabajo son su carácter retrospectivo, el sesgo de no incluir los casos diagnosticados en atención primaria y los posibles errores en el proceso de codificación de la fuente (informe de alta y/o historia clínica). Por último, el estudio no está actualizado debido al desfase entre la información de todos los centros de España y la generada en cada centro sanitario. No obstante, permite realizar estimaciones de la enfermedad en España.

En conclusión, la incidencia de la fiebre botonosa meditarránea durante el período 2009-2012 fue menor que en periodos anteriores, con una distribución heterogénea en zonas geográficas. El estudio sugiere que en España el perfil típico de la enfermedad es la de un varón adulto que enferma durante el verano y que se asocia a una baja mortalidad.

\section{AGRADECIMIENTOS}

A los equipos de documentación responsables del CMBD sin cuyas aportaciones no hubiera sido posible la realización de este trabajo.

\section{BIBLIOGRAFÍA}

1. Segura Porta F, Font Creus B. Fiebre botonosa mediterránea y otras infecciones causadas por rickettsias. Fiebre Q. En: Farreras P, Rozman C. Medicina Interna. $17^{\circ}$ ed. Barcelona: Elsevier; 2012.p.2107-2114.

2. Centro Nacional de Epidemiologia. Instituto de Salud Carlos III. Red Nacional de Vigilancia Epidemiológica. Protocolos de enfermedades de declaración obligatoria. Disponible en: http://www.isciii.es/ISCIII/es/contenidos/fd-servicios-cientifico-tecnicos/fd-vigilancias-alertas/PROTOCOLOS_RENAVE.pdf

3. Connor A, Bruch A: Une fièvre eruptive observée en Tunise. Bull Soc Pathol Exot Filial. 1910;8:492-6.

4. Brumpt E. Longevité du virus de la fièvre boutonneuse (Rickettsia conorii, n. sp.) chez la tique Rhipicephalus sanguineus. C R Soc Biol. 1932; 110:1197-9.

5. Boletín Oficial del Estado. Real Decreto 2210/1995 de 28 de diciembre, por el que se crea la Red Nacional de Vigilancia Epidemiológica. BOE núm 21 de 24/1/1996.
6. Gogorcena MA. Utilización del CMBD y estadísticas de hospitales del SNS. Escuela Nacional de Sanidad; 2013 [consultado el 1/02/2015]. Disponible en: http://e-spacio.uned.es/fez/eserv/bibliuned:500653/ n5.6_Utilizaci_n_del_CMBD.pdf

7. Instituto Nacional de Estadística. Disponible en http:// www.ine.es [Consultado el 2/02/2015].

8. Allúe M, Martín C. Zoonosis no alimentarias en Castilla y León. Boletín Epidemiológico de Castilla y León. 2007;23(5):29-36.

9. Punda-Polic V, Luksić B, Capkun V Epidemiological features of Mediterranean spotted fever, murine typhus, and Q fever in Split-Dalmatia County (Croatia), 19822002 Epidemiol Infect. 2008;136(07):972-979.

10. Baltadzhiev IG, Popivanova NI. Some epidemiological features of the Mediterranean spotted fever re-emerging in Bulgaria. Folia Med. 2012;54(1):36-43.

11. Rovery C, Brouqui P, Raoult D. Emerging questions on Mediterranean Spotted Fever one century after its discovery. Emerg Infect Dis. 2008;14:1360-7.

12 .García B, Colomina J, Borrás M, Guerrero A. Fiebre botonosa Mediterránea: Estimación de la incidencia en la Comunidad Valenciana durante el periodo 2009-2013. Libro de Abstracts del X Congreso de la Sociedad de Enfermedades Infecciosas de la Comunidad Valenciana y XXI Congreso de la Sociedad de Microbiología Clínica; 2015 abril 29-31: resúmenes de comunicaciones de la Sociedad de Enfermedades Infecciosas de la Comunidad Valenciana y Sociedad de Microbiología Clínica.

13. Guerrero A, Gimeno F, Colomina J, Molina M, Oteo JA, Cuenca M. Low incidence of tick-borne rickettsiosis in a Spanish Mediterranean area. Ann N Y Acad Sci 2006; 1078(1):200-202.

14. Centro Nacional de Epidemiologia. Resultados de la vigilancia epidemiológica de las enfermedades transmisibles. Informe anual 2009. Disponible en: http://www. isciii.es/ISCIII/es/contenidos/fd-servicios-cientifico-tecnicos/fd-vigilancias-alertas/fd-enfermedades/RENAVE_ INFORME_ANUAL_2009.pdf

15. Centro Nacional de Epidemiología. Instituto de Salud Carlos III. Resultados de la vigilancia epidemiológica de las enfermedades transmisibles. Informe anual 2010. Disponible en: http:/gesdoc.isciii.es/gesdoccontroller?a ction $=$ download $\&$ id $=21 / 01 / 2013-9221 \mathrm{~d} 7165 \mathrm{e}$

16. Centro Nacional de Epidemiología. Instituto de Salud Carlos III. Resultados de la vigilancia epidemiológica de las enfermedades transmisibles. Informe anual 2011. Disponible en: http:/gesdoc.isciii.es/gesdoccontroller?a ction=download\&id=14/11/2013-be6b0679b9 
17. Centro Nacional de Epidemiología. Resultados de la vigilancia epidemiológica de las enfermedades transmisibles. Informe anual 2012. Disponible en: http:// gesdoc.isciii.es/gesdoccontroller?action=download\& $\mathrm{id}=29 / 01 / 2015-\mathrm{f} 0855 \mathrm{cb} 161$

18. Parola P, Raoult D. Ticks and tickborne bacterial diseases in humans: an emerging infectious threat. Clin Infect Dis. 2001;32:897-928.

19. Bartolomé J, Lorente $\mathrm{S}$, Hernández-Pérez $\mathrm{N}$, Martínez-Alfaro E, Marín-Ors A, Crespo MD. Estudio clínico-epidemiológico de las rickettsiosis del grupo de las fiebres exantemáticas en Albacete. Enferm Infecc Microbiol Clin. 2005;23(4):194-6.

20. Pitigoi D, Olaru ID, Badescu D, Rafila A, Arama V, Hristea A. Mediterranean spotted fever in southeastern Romania. Biomed Res Int. 2013. 